\title{
Resilience, Anxiety, Stress, and Substance Use Patterns During COVID-19 Pandemic in the Miami Adult Studies on HIV (MASH) Cohort
}

\author{
Janet Diaz-Martinez ${ }^{1}$. Javier A. Tamargo ${ }^{1}$. Ivan Delgado-Enciso ${ }^{2}$ - Qingyun Liu' ${ }^{1}$. Leonardo Acuña ${ }^{3}$. \\ Eduardo Laverde ${ }^{3}$. Manuel A. Barbieri ${ }^{3} \cdot$ Mary Jo Trepka' ${ }^{1}$. Adriana Campa ${ }^{1}$ - Suzanne Siminski ${ }^{4}$. \\ Pamina M. Gorbach ${ }^{5} \cdot$ Marianna K. Baum ${ }^{1}$ (I)
}

Accepted: 25 April 2021 / Published online: 19 May 2021

(c) The Author(s), under exclusive licence to Springer Science+Business Media, LLC, part of Springer Nature 2021

\begin{abstract}
We evaluated mental health and substance use during the COVID-19 pandemic in 196 participants from the Miami Adult Studies on HIV (MASH) Cohort. A survey was administered between July-August of 2020, including validated measures of resilience and anxiety, a scale to measure COVID-19-related worry, and self-reported substance use. Compared to HIVuninfected participants $(n=80)$, those living with HIV $(n=116)$ reported fewer anxiety symptoms, less COVID-19-related worry, and higher resilience. Those with more anxiety symptoms and lower resilience engaged in more frequent alcohol consumption, binge drinking, and cocaine use. Alcohol misuse was more common among HIV-uninfected participants. Cocaine use was reported by $21 \%$ fewer participants during the pandemic compared with $7.3 \pm 1.5$ months earlier. Possibly due to their experiences with HIV, PLWH responded with higher resilience and reduced worry and anxiety to the adversities brought by the COVID-19 pandemic.
\end{abstract}

Keywords HIV · COVID-19 - Resilience $\cdot$ Mental health $\cdot$ Substance use

\section{Introduction}

The circumstances generated by the COVID-19 pandemic and the response to mitigate the spread of the infection have had immense psychological impacts on mental health on a global scale $[1,2]$. A recent systematic review and meta-analysis concluded that the COVID-19 pandemic can increase the prevalence of stress, anxiety, and depression in the general population [2]. COVID-19 could impose greater psychological effects on vulnerable communities affected

Marianna K. Baum

baumm@fiu.edu

Robert Stempel College of Public Health, Florida International University, Miami, FL, USA

2 School of Medicine, University of Colima, Colima, MX, Mexico

3 College of Arts, Sciences \& Education, Florida International University, Miami, FL, USA

4 Frontier Science Foundation, Brookline, MA, USA

5 Fielding School of Public Health, University of California, Los Angeles, CA, USA by health disparities $[3,4]$. The pandemic may particularly adversely affect people living with HIV (PLWH) who are already disproportionately impacted by racial and psychosocial hardships, including stigma and isolation [5]. In addition, PLWH suffer from high rates of multiple comorbidities including diabetes, hypertension, liver disease, and elevated rates of alcohol and substance use disorders (SUDs) [6-9].

On the other hand, PLWH are considered a resilient population [10]. Resilience has been described as a dynamic process influenced by biological, social, and environmental factors [11]. In a systematic review of literature, Windle defined resilience as "the process of effectively negotiating, adapting to, or managing significant sources of stress or trauma" [12]. Resilience may ameliorate psychological distress after traumatic events [13]. For example, higher levels of resilience were associated with lower anxiety and depression among COVID-19 patients in Wuhan, China [14]. Nonetheless, the psychological status of PLWH and their ability to cope during the COVID-19 pandemic has not yet been sufficiently explored.

Limited scientific evidence is available on how the COVID-19 pandemic could lead to changes in substance use patterns [15], which could ultimately heighten the 
risks for the infection and lead to adverse outcomes. The National Institute on Drug Abuse (NIDA) has declared that the COVID-19 pandemic presents unique challenges for people with SUDs, such as interfering with recovery. NIDA, therefore, has urged the research community to collect and share data on drug use trends and their relationship with COVID-19 [16].

South Florida suffers from multiple epidemics. In addition to the current COVID-19 crisis, South Florida has one of the highest HIV incidence rates in the nation [17], an epidemic that has been strongly linked to drug use in Miami, Florida [18-20]. The clinical impact of HIV infection on the natural history of COVID-19 is uncertain, although limited evidence suggests that PLWH are not at greater risk of infection, severe disease, or death than the general population, if they are effectively treated with antiretroviral therapy (ART) $[21,22]$. Therefore, the impact of the COVID-19 epidemic on vulnerable and marginalized communities, and the intricate relationship between mental health and drug use in the context of HIV and the COVID-19 pandemic warrants examination. The objectives of this study were to evaluate resilience, mental health and substance use patterns during the COVID-19 pandemic among people living with and without HIV from the Miami Adult Studies on HIV (MASH) Cohort.

\section{Methods}

Data was collected during July and August of 2020 from participants in the Miami Adult Studies on HIV (MASH) cohort, which follows over 1000 people living with and without HIV. The MASH cohort is largely comprised of Black and Hispanic, middle-aged individuals who live in a poor urban sector of Miami, FL. Participants are recruited from neighboring clinics, particularly the Borinquen Health Care Center (https://www.borinquenhealth.org/), as well as nearby food banks and shelters. Participation in the MASH cohort is contingent on confirmation of HIV status (e.g. rapid antigen/antibody test, nucleic acid test) confirmed with name and date of birth or from medical records with participants' signed release of medical information. Participants are followed every 6 months for a range of data, including morbidity, healthcare access and utilization, medication adherence, socioeconomic factors, and use of alcohol, tobacco, and illicit substances.

All MASH cohort participants were eligible to participate in the survey. A convenience sample of $200 \mathrm{MASH}$ cohort participants were randomly contacted by telephone and invited to participate in a supplemental study of the impact of the COVID-19 epidemic on their lives. In total, 232 participants were called, 200 were reached, and all of those that were reached agreed to participate. Four participants were excluded from the analyses due to missing data.
The study protocol was approved by the Institutional Review Board at Florida International University. Verbal consent was obtained, witnessed, and documented by two members of our research staff. A survey was administered by phone and participants were given an incentive of \$20 for their participation. The survey was designed by the Collaborating Consortium of Cohorts Producing NIDA Opportunities (C3PNO), which coordinates U01 cohort studies funded by the National Institutes for Drug Abuse (NIDA). The C3PNO COVID-19 Survey includes original and validated questionnaires; survey and sources available at https://www.c3pno. org.

\section{Data from the COVID-19 Survey}

The General Anxiety Disorder-7 (GAD-7) is a 7-item screening tool used to assess symptoms of anxiety [23, 24]. The total score ranges from 0 to 21 ; scores of 10 or more are indicative of the presence of anxiety symptoms.

COVID-19-related worry was assessed by asking participants "On a scale of 1 to 10 , how worried are you about the COVID-19 pandemic? 1 being not worried at all and 10 being extremely worried." Scores of $\geq 6$ were considered high levels of worry.

The Brief Resilience Scale (BRS) was used to assess resilience $[25,26]$. Scores range from 0 to 6 . High resilience was defined as scores above the median value for our study sample (>3.4).

The Alcohol Use Disorder Identification Test-Consumption (AUDIT-C) questionnaire was used to assess alcohol consumption [27]. Scores of $\geq 4$ for men and $\geq 3$ for women are indicative of alcohol misuse. Binge drinking was determined with the third item of the AUDIT-C and defined as "monthly or more" consumption of $\geq 5$ or $\geq 4$ drinks on one occasion for males and females, respectively.

\section{Data Collected Pre-pandemic}

Data from participants' last cohort visit was used to supplement this analysis, with a mean range of $7.3 \pm 1.5$ months between the two assessments. Sociodemographic characteristics (age, gender, race/ethnicity, income), AUDIT-C, and substance use (30-day frequency) were self-reported. Anthropometric and blood pressure measurements and fasting blood draws were performed by trained research staff and used to determine body mass index (BMI), hypertension (systolic $\geq 130$ or diastolic $\geq 85 \mathrm{~mm} \mathrm{Hg}$ ), and hyperglycemia (fasting glucose $\geq 100 \mathrm{mg} / \mathrm{dL}$ ).

\section{Statistical Analysis}

Descriptive statistics are reported as mean \pm standard deviation (SD) or as No. (\%). The primary independent variable 
was HIV status, and the primary outcomes were anxiety symptoms, COVID-19-related worry, resilience, and drug use. T-tests and Chi square tests were performed to test for group differences in continuous and categorical outcomes, respectively. Within group differences in frequencies were tested with McNemar's test. Correlation analyses included Pearson product-moment correlations for two continuous variables and Spearman's rank correlations when at least one variable was ordinal. Logistic regressions were performed to calculate odds ratios (OR) and $95 \%$ confidence intervals (CI) for anxiety symptoms, high levels of COVID-19-related worry, and high resilience for PLWH compared to HIVuninfected participants. Adjusted odds ratios (AOR) were calculated by adjusting for sociodemographic characteristics (age, sex, race/ethnicity, income, education, housing, and employment). Results were considered statistically significant at two-tailed $P<0.05$. The data analysis for this paper was generated using SAS software, version 9.4. Copyright (C) 2002-2012 SAS Institute Inc.

\section{Results}

\section{Sample Characteristics}

Table 1 displays the socioeconomic and clinical characteristics of the participants. The study sample included a total of 196 participants, including 116 (59.2\%) PLWH and 80 (40.8\%) HIV-uninfected participants. A total of 69 (35.2\%) participants reported having been tested for COVID-19 by nasopharyngeal swabs and only $4(2.0 \%)$ had tested positive. Participants had a mean age of $56.6 \pm 6.7,51.0 \%$ were female, $75.5 \%$ Black non-Hispanic, and $16.8 \%$ Hispanic. The vast majority earned below $\$ 15,000$ annually per household (76.3\%), were unemployed (including disability, 90.3\%), and had a high-school education or less $(72.4 \%)$. Prior to the pandemic, most participants reported having health insurance $(87.2 \%)$, Medicare or Medicaid coverage (80.5\%), and had received primary care in the past 12 months (93.3\%), but less than half (47.7\%) reported having received mental health care. Only $13(6.6 \%)$ participants reported having health insurance without Medicare or Medicaid coverage.

The majority of PLWH were receiving ART (99.1\%), $86.2 \%$ were virally suppressed ( $<200$ HIV RNA copies $/ \mathrm{mL}$ ) and had a mean CD4 lymphocyte count of $652.7 \pm 333.9$ cells $/ \mu \mathrm{L}$ at their last clinic visit. Compared to PLWH, HIVuninfected participants were slightly older $(55.7 \pm 6.5$ vs. $57.8 \pm 6.8$, respectively; $t=2.16, P=0.032)$ and were more likely to be homeless ( $2.6 \%$ vs. $10.0 \%$, respectively; $\left.\chi^{2}=4.91, P=0.026\right)$. Additionally, prior to the pandemic, a greater proportion of PLWH reported having health insurance $\left(92.1 \%\right.$ vs. $\left.80.3 \% ; \chi^{2}=5.96, P=0.015\right)$, Medicare or Medicaid coverage $\left(85.1 \%\right.$ vs. $\left.74.1 \% ; \chi^{2}=3.66, P=0.056\right)$, and receiving primary $\left(97.4 \%\right.$ vs. $87.7 \% ; \chi^{2}=7.18$, $P=0.007)$ and mental health care $(51.8 \%$ vs. $42.0 \%$; $\left.\chi^{2}=1.82, P=0.178\right)$ in the past 12 months, although the difference was only statistically significant for insurance and primary care. No other sociodemographic or clinical characteristic significantly differed between PLWH and HIVuninfected participants.

The characteristics of the participants in this study were representative of the overall characteristics of the MASH cohort, with the exception that, compared to the overall cohort, Hispanics were under-sampled $(16.8 \%$ vs. $29.0 \%$, respectively) and Black non-Hispanics were over-sampled (75.5\% vs. $61.7 \%$, respectively).

\section{Mental Health and Substance Use}

We performed correlation analyses between GAD-7, COVID-19-related worry, BRS, and substance use (Table 2). Anxiety symptoms were directly correlated with COVID19-related worry $(r=0.33, P<0.0001)$, and these were both inversely correlated with resilience $(r=-0.40$ and $r=-0.14$, respectively; $P<0.0001$ for both). Additionally, anxiety symptoms correlated with alcohol misuse, binge drinking, and cocaine use. Resilience was inversely correlated with binge drinking.

Stratifying the sample by HIV status showed several differences related to substance use patterns. Among PLWH, anxiety symptoms directly correlated with binge drinking and cocaine use. On the other hand, among HIV-uninfected participants, COVID-19-related worry correlated with alcohol misuse.

\section{Resilience, Anxiety, and Stress}

Anxiety symptoms, COVID-19-related worry, and high resilience were reported by $18.9 \%, 71.4 \%$ and $49 \%$ of participants, respectively. PLWH, compared to HIV-uninfected participants, had lower mean GAD-7 (measure for anxiety) $(3.6 \pm 5.1$ vs $6.8 \pm 6.2 ; t=3.92, P<0.001)$ and COVID19-related worry $(6.8 \pm 3.2$ vs $7.9 \pm 3.0 ; t=2.30, P=0.023)$, and higher BRS (measure for resilience) $(3.5 \pm 0.6$ vs $3.3 \pm 0.7 ; t=2.17, P=0.031$ ), respectively (Table 3 ). Similar results were obtained when comparing the risk for anxiety symptoms, high levels of COVID-19-related worry, and high resilience (Table 3). Compared to HIV-uninfected participants, PLWH had lower odds of having anxiety symptoms $\left(\mathrm{OR}=0.39,95 \%\right.$ CI $\left.0.18-0.81 ; \chi^{2}=6.31, P=0.012\right)$ and lower odds of high levels of COVID-19-related worry $\left(\mathrm{OR}=0.42,95 \%\right.$ CI $\left.0.21-0.83 ; \chi^{2}=6.21, P=0.013\right)$, as well as twofold higher odds of high resilience $(\mathrm{OR}=2.01$ 95\% CI 1.13-3.60; $\left.\chi^{2}=5.59, P=0.018\right)$. These relationships remained largely unchanged after adjusting for 
Table 1 Socioeconomic and clinical characteristics of MASH cohort participants during the COVID-19 pandemic

\begin{tabular}{|c|c|c|c|c|c|}
\hline & $\begin{array}{l}\text { Total } \\
(\mathrm{N}=196)\end{array}$ & $\begin{array}{l}\mathrm{HIV+} \\
(\mathrm{N}=116)\end{array}$ & $\begin{array}{l}\text { HIV- } \\
(\mathrm{N}=80)\end{array}$ & Test & $P$ \\
\hline & $\begin{array}{l}\% \text { or } \\
\text { Mean } \pm \text { SD }\end{array}$ & $\begin{array}{l}\% \text { or } \\
\text { Mean } \pm \text { SD }\end{array}$ & $\begin{array}{l}\% \text { or } \\
\text { Mean } \pm \text { SD }\end{array}$ & & \\
\hline Age, years & $56.6 \pm 6.7$ & $55.7 \pm 6.5$ & $57.8 \pm 6.8$ & $t=2.16$ & 0.032 \\
\hline Sex, male & $49.0 \%$ & $50.0 \%$ & $47.5 \%$ & $\chi^{2}=0.118$ & 0.730 \\
\hline \multicolumn{6}{|l|}{ Race/ethnicity } \\
\hline Black non-Hispanic & $75.5 \%$ & $77.6 \%$ & $72.5 \%$ & \multirow[t]{3}{*}{$\chi^{2}=2.07$} & \multirow[t]{3}{*}{0.358} \\
\hline White non-Hispanic & $7.7 \%$ & $8.6 \%$ & $6.2 \%$ & & \\
\hline Hispanic & $16.8 \%$ & $13.8 \%$ & $21.2 \%$ & & \\
\hline \multicolumn{6}{|l|}{ Household income } \\
\hline$\$ 15,000$ or less & $76.3 \%$ & $74.1 \%$ & $79.7 \%$ & \multirow[t]{3}{*}{$\chi^{2}=3.86$} & \multirow[t]{3}{*}{0.143} \\
\hline$\$ 15,000-\$ 30,000$ & $20.4 \%$ & $24.1 \%$ & $14.9 \%$ & & \\
\hline$\$ 30,000$ or more & $3.2 \%$ & $1.8 \%$ & $5.4 \%$ & & \\
\hline \multicolumn{6}{|l|}{ Education } \\
\hline Less than high-school & $45.9 \%$ & $46.6 \%$ & $45.0 \%$ & \multirow[t]{3}{*}{$\chi^{2}=0.099$} & \multirow[t]{3}{*}{0.951} \\
\hline High-school or GED & $26.5 \%$ & $26.7 \%$ & $26.3 \%$ & & \\
\hline More than high-school & $27.6 \%$ & $26.7 \%$ & $28.7 \%$ & & \\
\hline \multicolumn{6}{|l|}{ Housing } \\
\hline House/apartment & $94.4 \%$ & $97.4 \%$ & $90.0 \%$ & \multirow[t]{2}{*}{$\chi^{2}=4.91$} & \multirow[t]{2}{*}{0.026} \\
\hline Homeless & $5.6 \%$ & $2.6 \%$ & $10.0 \%$ & & \\
\hline \multicolumn{6}{|l|}{ Employment } \\
\hline Unemployed & $90.3 \%$ & $93.1 \%$ & $86.3 \%$ & \multirow[t]{2}{*}{$\chi^{2}=2.54$} & \multirow[t]{2}{*}{0.111} \\
\hline Employed & $9.7 \%$ & $6.9 \%$ & $13.7 \%$ & & \\
\hline \multicolumn{6}{|l|}{ Healthcare } \\
\hline Insured & $87.2 \%$ & $92.1 \%$ & $80.3 \%$ & $\chi^{2}=5.96$ & 0.015 \\
\hline Medicare or medicaid & $80.5 \%$ & $85.1 \%$ & $74.1 \%$ & $\chi^{2}=3.66$ & 0.056 \\
\hline Received primary care ${ }^{\mathrm{a}}$ & $93.3 \%$ & $97.4 \%$ & $87.7 \%$ & $\chi^{2}=7.18$ & 0.007 \\
\hline Received mental care ${ }^{\mathrm{b}}$ & $47.7 \%$ & $51.8 \%$ & $42.0 \%$ & $\chi^{2}=1.82$ & 0.178 \\
\hline \multicolumn{6}{|l|}{ Metabolic } \\
\hline Obesity & $45.9 \%$ & $46.6 \%$ & $45.0 \%$ & $\chi^{2}=0.046$ & 0.830 \\
\hline Hypertension (SBP $\geq 130$ OR DBP $\geq 85$ ) & $44.9 \%$ & $44.9 \%$ & $45.0 \%$ & $\chi^{2}=0.001$ & 0.981 \\
\hline Hyperglycemia (Fasting glucose $\geq 100$ ) & $19.9 \%$ & $16.5 \%$ & $25.0 \%$ & $\chi^{2}=2.12$ & 0.145 \\
\hline
\end{tabular}

Data are presented as the mean \pm standard deviation (using $t$ test for comparison) and percentages (using Pearson Chi square test for comparison). Bolded values denote statistical significance $(P<0.05)$.

${ }^{\text {a }}$ During the past 12 months, have you seen or talked to any of the following health care providers about your own health? A primary care physician or general physician (M.D. or D.O.)

${ }^{b}$ During the past 12 months, have you seen or talked to any of the following health care providers about your own health? A mental health professional such as a psychiatrist, psychologist, psychiatric nurse, or clinical social worker sociodemographic factors (age, gender, education, employment, income, housing status) and metabolic parameters (Table 3).

Additionally, we performed linear regressions to examine whether resilience confounded the relationship between HIV and anxiety symptoms and COVID-19-related worry (Table 4). In univariate regressions (Model 1), both HIV and resilience were independently associated with lower anxiety symptoms and COVID-19-related worry. When the model included both HIV and resilience, the effect of HIV on anxiety symptoms was attenuated, with a tendency to attenuate the effect of HIV on COVID-19-related worry as well (Model 2). On the other hand, we found no evidence of a significant interaction effect between HIV and resilience (Model 3).

\section{Substance Use Patterns}

Table 5 compares substance use between PLWH and HIVuninfected participants before and during COVID-19 pandemic. Before the COVID-19 pandemic, HIV-uninfected participants were more likely to use cocaine than PLWH 
Table 2 Correlation coefficient values among mental health scores and substance use during the COVID-19 pandemic in the Miami Adult HIV Studies (MASH) cohort

\begin{tabular}{|c|c|c|c|c|c|c|c|c|c|}
\hline & \multicolumn{3}{|c|}{ Total $(\mathrm{N}=196)$} & \multicolumn{3}{|c|}{ PLWH $(\mathrm{N}=116)$} & \multicolumn{3}{|c|}{ HIV-uninfected $(\mathrm{N}=80)$} \\
\hline & GAD-7 & Worry & BRS & GAD-7 & Worry & BRS & GAD-7 & Worry & BRS \\
\hline GAD- $7^{\mathrm{a}}$ & - & $0.33^{* * * * *}$ & $-0.40^{* * * * *}$ & - & $\mathbf{0 . 3 1} 1^{* * * *}$ & $-0.31^{* * * * *}$ & - & $0.30^{* * *}$ & $-0.46^{* * * * *}$ \\
\hline COVID-19 worry $^{\mathrm{a}}$ & - & - & $-0.14^{* * * * *}$ & - & - & -0.02 & - & - & $-0.26^{* * * * *}$ \\
\hline Tobacco $^{\mathrm{a}}$ & 0.09 & 0.06 & 0.01 & 0.14 & 0.05 & 0.02 & -0.03 & 0.02 & 0.04 \\
\hline Alcohol misuse ${ }^{b}$ & $0.16 *$ & -0.03 & 0.06 & 0.09 & 0.09 & 0.03 & 0.13 & $0.30^{* * *}$ & 0.19 \\
\hline Binge drinking ${ }^{\mathrm{b}}$ & $0.43^{* * * *}$ & 0.09 & $-0.35^{* *}$ & $0.32^{* *}$ & 0.20 & $-0.27^{\wedge}$ & $0.54^{* * * *}$ & 0.01 & $-0.41^{* *}$ \\
\hline Cannabis $^{\mathrm{b}}$ & 0.12 & -0.05 & -0.08 & 0.11 & 0.01 & -0.11 & 0.03 & -0.21 & -0.001 \\
\hline Cocaine $^{\mathrm{b}}$ & $0.19^{* *}$ & -0.06 & 0.05 & $0.24^{* *}$ & -0.10 & 0.03 & -0.13 & 0.03 & 0.04 \\
\hline
\end{tabular}

$B R S$ Brief Resilience Scale, GAD-7 General Anxiety Disorder-7, PLWH people living with HIV

Bolded values denote statistical significance $(P<0.05)$

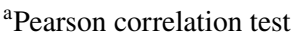

${ }^{\mathrm{b}}$ Spearman correlation test

$* \mathrm{P}$ value $<0.05 ; * * \mathrm{P}$ value $<0.01 ; * * * \mathrm{P}$ value $<0.001 ; * * * * \mathrm{P}$ value $<0.0001$

${ }^{\wedge}$ Tendency to statistical significance, $\mathrm{P}$ value $<0.10$ for a two-sided test

( $41.2 \%$ vs. $\left.26.7 \% ; \chi^{2}=4.54, P=0.033\right)$ and showed a trend towards higher alcohol misuse as well (43.7\% vs. $30.4 \%$; $\left.\chi^{2}=3.64, P=0.056\right)$. During the pandemic, there was a higher proportion of HIV-uninfected participants who reported alcohol misuse compared to PLWH $(50.0 \%$ vs $\left.29.3 \% ; \chi^{2}=8.62, P=0.003\right)$, but there was no difference in rates of cocaine use $\left(15.0 \%\right.$ vs. $\left.13.8 \% ; \chi^{2}=0.06, P=0.8\right)$. In fact, the frequencies of cocaine use significantly dropped by $12.9 \%$ and $26.5 \%$ among PLWH and HIV-uninfected participants, respectively. No between- or within-group differences were seen for cigarette smoking and use of cannabis.

When participants were asked "What have you noticed about the price of (cocaine) in the past month?" 19 (9.7\%) reported that prices of cocaine had gone up. When asked "What changes, if any, have you noticed regarding your ability to get (cocaine) in the past month?" 14 (7.14\%) reported that cocaine was harder to obtain.

\section{Discussion}

The COVID-19 pandemic generated a global crisis and a rapid implementation of extraordinary changes that have disproportionally impacted marginalized populations [28]. The aim of this study was to examine resilience, mental health and substance use patterns during the COVID-19 pandemic among people living with and without HIV from the MASH cohort. The data used in this analysis was collected between July and August of 2020, in the midst of rising COVID19 cases and deaths in Florida after easing of restrictions that were placed to mitigate the spread of the disease. Our findings revealed that PLWH reported higher resilience and less anxiety symptoms and COVID-19-related worry than
HIV-uninfected peers. Our findings also suggest that higher resilience partially explains why PLWH displayed lower anxiety and worry. Significant relationships were found between mental health, particularly anxiety, and substance use. Our results indicate that, compared to their peers, many PLWH may be protected from psychosocial stressors during the COVID-19 pandemic via psychological resilience, which they have built over time through the management of the HIV infection [10]. Our study emphasizes that public health efforts to promote resilience in vulnerable populations may enable such groups to cope with and overcome the adversities generated by the COVID-19 pandemic.

Resilience can be considered a positive adaptation to challenges and is comprised of both internal (e.g. self-efficacy, coping skills) and external factors (e.g. social support). Our findings suggest that PLWH have higher resilience than HIV-uninfected peers. People living with HIV, due to their effort to manage a chronic and potentially fatal disease, have developed resilient characteristics [10]. This, in turn, might have equipped PLWH with coping mechanisms that allow them to adapt and manage their anxiety and worry about the COVID-19 pandemic to a greater degree than individuals with the same race/ethnicity and similar socioeconomic status who are uninfected with HIV. PLWH have been living with HIV for an average of $16 \pm 9$ years and are highly engaged in HIV care and are adherent to treatment, as evidenced by the great majority of them (86.2\%) having suppressed HIV viral load. As such, the resilience observed among PLWH in this study may be a result of their many years living with and managing HIV disease, as well as their unfettered access to medical care and support services.

Resilience was inversely correlated with anxiety and worry. Furthermore, only $13 \%$ of PLWH reported anxiety 
symptoms as compared to $28 \%$ of the HIV-uninfected participants. In comparison, a population-based study of PLWH reported a $19 \%$ prevalence of anxiety symptoms during 2015-2016, also using the GAD-7 [29]. Among the general U.S. population, the rate of anxiety reported in 2019 was $8.2 \%$ and rose to approximately $30 \%$ during the COVID-19 pandemic [30], similar to the rate observed among HIV-uninfected participants in the MASH cohort. Likewise, the proportion of participants who reported high levels of COVID-19-related worry was significantly lower among PLWH (64.7\%) than among the HIV-uninfected participants $(81.3 \%)$. Higher resilience among PLWH may in part explain the lower prevalence of anxiety symptoms and COVID-19 related worry when compared to HIV-uninfected participants. Indeed, resilience attenuated the effect of HIV status on mental health, particularly anxiety symptoms. These findings are consistent with prior reports. A large multicenter study of PLWH in the United Kingdom found that resilience was inversely related to the prevalence of depression and anxiety [31]. Resilience has been found to be a protective factor for the mental health of HIV + men who have sex with men $[32,33]$.

Additionally, our study shows that the COVID-19 pandemic has impacted substance use in the MASH cohort. Cocaine use is a major cause of drug-related deaths in South Florida [34]; yet, we found a significant decline in its use during the COVID-19 pandemic as compared to $7.3 \pm 1.5$ months earlier, prior to lockdowns. While cocaine use was higher among HIV-uninfected participants than PLWH prior to the pandemic, the frequency of use during the pandemic did not differ between the two groups. We did not observe significant changes in alcohol consumption, but alcohol misuse was more prevalent among the HIV-uninfected participants during the pandemic. Interestingly, the prevalence of alcohol misuse during the pandemic among PLWH was $29 \%$, similar to what has been reported for PLWH in the U.S. before the pandemic (27\%) [35].

The reduction of cocaine use follows a reduction in the supply chain of the drug, imposed by the measures to contain the pandemic, rather than individual decisions or circumstances [36, 37]. In fact, the participants reported that the drug had become more expensive and harder to obtain. During the pandemic, cocaine prices have sharply increased in Miami [36], and $76.3 \%$ of our study population earned $\$ 15,000$ or less annually, which made the existing illicit market too expensive to sustain with their income. A report prepared by the United Nations Office on Drug Crime Global Research Network showed that new patterns of drug use have emerged during the pandemic [37]. While there has been an overall decrease in consumption of drugs, there are concerns about increased use of more easily obtained substances (e.g. alcohol) and more harmful patterns of drug use among people with SUDs [15, 37]. 
Table 4 Resilience as a potential confounder between HIV and mental health

Table 5 Comparison of substance use by HIV status and time of assessment (before and during the COVID-19 pandemic)

\begin{tabular}{|c|c|c|c|c|c|c|c|c|c|}
\hline \multirow[t]{2}{*}{ Model } & \multirow[t]{2}{*}{ Parameter } & \multicolumn{4}{|c|}{ Anxiety symptoms } & \multicolumn{4}{|c|}{ COVID-19 worry } \\
\hline & & $\beta$ & SE & $t$ & $P$ & $\beta$ & SE & $t$ & $P$ \\
\hline 1a (univariate) & HIV & -3.17 & 0.81 & -3.92 & 0.0001 & -1.05 & 0.46 & -2.30 & 0.023 \\
\hline 1b (univariate) & Resilience & -3.39 & 0.55 & -6.14 & $<0.0001$ & -0.66 & 0.33 & -2.01 & 0.046 \\
\hline \multirow[t]{2}{*}{2 (multiple) } & HIV & -2.50 & 0.76 & -3.30 & 0.001 & -0.93 & 0.46 & -2.02 & 0.045 \\
\hline & Resilience & -3.11 & 0.54 & -5.72 & $<0.0001$ & -0.56 & 0.33 & -1.69 & 0.093 \\
\hline \multirow[t]{3}{*}{3 (with interaction) } & HIV & -8.29 & 3.81 & -2.18 & $\mathbf{0 . 0 3 1}$ & -4.37 & 2.31 & -1.89 & 0.060 \\
\hline & Resilience & -0.67 & 1.66 & -0.40 & 0.687 & -0.89 & 1.01 & -0.88 & 0.378 \\
\hline & HIV*Resilience & -1.70 & 1.09 & -1.55 & 0.123 & -1.01 & 0.66 & -1.52 & 0.131 \\
\hline
\end{tabular}

${ }^{a}$ Resilience was measured with the Brief Resilience Scale (BRS); anxiety symptoms were measured with the Generalized Anxiety Disorder-7 (GAD-7); COVID-19-related worry was measured with a scale of 1 to 10

Bolded values denote statistical significance $(P<0.05)$

\begin{tabular}{|c|c|c|c|c|c|c|}
\hline Substances & & $\begin{array}{l}\text { Total } \\
\mathrm{N}=196\end{array}$ & $\begin{array}{l}\text { PLWH } \\
\mathrm{N}=116\end{array}$ & $\begin{array}{l}\text { HIV-uninfected } \\
\mathrm{N}=80\end{array}$ & $\chi^{2}$ & $P^{\mathrm{a}}$ \\
\hline \multirow[t]{4}{*}{ Alcohol misuse ${ }^{c}$} & Before & $35.9 \%$ & $30.4 \%$ & $43.7 \%$ & 3.64 & 0.056 \\
\hline & $\begin{array}{l}\text { During } \\
\text { COVID-19 }\end{array}$ & $37.8 \%$ & $29.3 \%$ & $50.0 \%$ & 8.62 & 0.003 \\
\hline & $\chi^{2}$ & 0.24 & 0.22 & 1.32 & & \\
\hline & $P^{\mathbf{b}}$ & 0.622 & 0.637 & 0.251 & & \\
\hline \multirow[t]{4}{*}{ Binge drinking ${ }^{\mathrm{d}}$} & Before & $17.3 \%$ & $14.8 \%$ & $21.3 \%$ & 1.37 & 0.242 \\
\hline & $\begin{array}{l}\text { During } \\
\text { COVID-19 }\end{array}$ & $15.8 \%$ & $12.9 \%$ & $20.0 \%$ & 1.78 & 0.183 \\
\hline & $\chi^{2}$ & 0.31 & 0.33 & 0.06 & & \\
\hline & $P^{\mathbf{b}}$ & 0.578 & 0.564 & 0.808 & & \\
\hline \multirow[t]{4}{*}{ Smoker (tobacco) } & Before & $51.0 \%$ & $47.4 \%$ & $56.2 \%$ & 1.48 & 0.223 \\
\hline & $\begin{array}{l}\text { During } \\
\text { COVID-19 }\end{array}$ & $48.0 \%$ & $43.1 \%$ & $55.0 \%$ & 2.68 & 0.101 \\
\hline & $\chi^{2}$ & 1.64 & 2.78 & 0.08 & & \\
\hline & $P^{\mathbf{b}}$ & 0.201 & 0.096 & 0.782 & & \\
\hline \multirow[t]{4}{*}{ Cannabis } & Before & $26.0 \%$ & $23.3 \%$ & $30.0 \%$ & 1.11 & 0.291 \\
\hline & $\begin{array}{l}\text { During } \\
\text { COVID-19 }\end{array}$ & $25.5 \%$ & $21.5 \%$ & $31.2 \%$ & 2.34 & 0.125 \\
\hline & $\chi^{2}$ & 0.07 & 0.40 & 0.20 & & \\
\hline & $P^{\mathbf{b}}$ & 0.796 & 0.5271 & 0.6547 & & \\
\hline \multirow[t]{4}{*}{ Cocaine } & Before & $32.65 \%$ & $26.7 \%$ & $41.2 \%$ & 4.54 & 0.033 \\
\hline & $\begin{array}{l}\text { During } \\
\text { COVID-19 }\end{array}$ & $14.3 \%$ & $13.8 \%$ & $15.0 \%$ & 0.06 & 0.812 \\
\hline & $\chi^{2}$ & 36.00 & 15.00 & 21.00 & & \\
\hline & $P^{\mathbf{b}}$ & $<0.001$ & 0.0001 & $<0.001$ & & \\
\hline
\end{tabular}

AUDIT-C Alcohol Use Disorders Identification Test-Consumption, $P L W H$ people living with HIV

Bolded values denote statistical significance $(P<0.05)$

${ }^{\mathrm{a}}$ Chi square tests were performed for between groups comparisons of PLWH vs. HIV-uninfected

${ }^{b}$ McNemar's tests were performed for within group comparisons of before vs. during COVID-19 pandemic

${ }^{\mathrm{c}}$ Alcohol misuse was determined by AUDIT-C $\geq 4$ in men, $\geq 3$ in women

${ }^{\mathrm{d}}$ Binge drinking was determined by self-reported monthly or higher consumption of $\geq 5$ or $\geq 4$ drinks on one occasion for males and females, respectively, on the AUDIT- 3 
When we examined the relationship between resilience, mental health, and substance use during the pandemic, we found that anxiety symptoms correlated with alcohol misuse, binge drinking, and cocaine use. Resilience was inversely correlated with binge drinking. COVID-19-related worry was correlated with alcohol misuse only among HIV-uninfected participants. Mental health disorders, such as mood and anxiety disorders, often co-occur with SUDs [38-40]. In the general U.S. adult population, almost $20 \%$ of all persons with a diagnosis of SUD had at least one co-occurring anxiety disorder [38]. These connections underscore the crucial role of mental health in the successful treatment of SUDs and in preparing and supporting populations for dealing with crises, such as pandemics and natural and man-made disasters. Some have called for a larger emphasis on resilience in the treatment of SUDs, as it may have behavioral and biological benefits [41]. Because psychological resilience may offer a level of protection against mental health disorders $[42,43]$, strategies to promote resilience at the individual and community levels may offer substantial benefits during and after the COVID-19 pandemic.

Chandra et al. described psychological (and physical) health as one of the core components of community resilience [44] and how health promotion is a key lever to enhance community resilience [45]. Not surprisingly, PLWH in the MASH cohort report healthcare access and utilization in greater proportions than HIV-uninfected participants. Federally-funded programs like the Ryan White HIV/AIDS Program support PLWH with insufficient financial resources with medical and support services including mental health and treatment for SUDs. This type of support may contribute to resilience among PLWH and may partially explain the results of this study. Consequently, increased access to mental health and substance use services could play a role in increasing resilience, mitigating anxiety and stress, and addressing social needs that may arise during the COVID-19 pandemic among HIV-uninfected people who may not have access to medical care and other support services. While the COVID-19 pandemic has presented with significant challenges to public mental health, it is also an opportunity for improvements in mental health services [46]. To mitigate the impact of the pandemic, health systems should facilitate the continuation of mental health services for those with pre-existing conditions, as well as increase access to those who develop mental health problems during the pandemic whether they are HIV-infected or not [46]. Community outreach, through partnerships between healthcare providers and other social services, can help promote mental health literacy, identify stressors in the community, and encourage vulnerable individuals to seek care [46].

Community resilience is considered fundamental to the preparedness, response, and recovery to major traumatic events [47]. In order to achieve community resilience, social, economic, structural, and health inequities must be addressed. Targeted strategies to enhance and strengthen individual and community resilience may help vulnerable populations to adapt to the challenges of the COVID-19 pandemic and aid in their post-pandemic recovery. In addition to health promotion, Chandra et al. delineate education as a way to improve risk communication $[44,45]$. Tailored educational materials can raise awareness, encourage protective behaviors, and relieve stress and anxiety by informing and empowering the community. The integration and collaboration of governmental and non-governmental entities are essential to building and strengthening community resilience [44] and build trust with minorities that often mistrust government and medical systems [48-50]. Moreover, the relationships between organizations and individuals can play a significant role in building social connectedness, further contributing to the resilience of a community [44].

Several strengths and limitations of our study must be acknowledged. Our study was conducted in a well-characterized cohort of an underserved and vulnerable population living in South Florida, largely comprised of low-income minorities living with and without HIV and high rates of comorbidities. As mentioned earlier, PLWH in the MASH cohort have been living with HIV for nearly two decades on average and are highly engaged with HIV care. Subsequently, our findings may not be generalizable to some groups of PLWH within the U.S., such as those recently diagnosed and those that may not be receiving treatment. On the other hand, the MASH cohort resembles a large portion of the U.S. population of PLWH in that they are on average middle-aged, Black non-Hispanic, and receiving HIV care [17, 51]. The HIV-uninfected participants from the MASH cohort were recruited from the same community as their HIV + peers, therefore representing middle-aged, low-income minorities with several social and economic disadvantages. Validated and frequently used measures of resilience and anxiety symptoms were used, allowing for comparisons to other communities and populations. However, the data was self-reported, thus subject to bias and under-reporting of substance use. Nonetheless, surveys were administered by trained research staff working with these participants every 6 months, which limits potential misunderstanding of the questions from the respondents. Lastly, the cross-sectional study design does not allow for causation to be inferred. Thus, longitudinal follow-up of the MASH cohort based on this study's findings may help us identify the unique social disparities and mental health challenges of a highly vulnerable community and inform public health practices to improve mental health relief during times of crises. The dissemination of our results can increase the public visibility and awareness of the mental health vulnerabilities faced by marginalized communities amid the 
COVID-19 pandemic and unify efforts to eliminate mental health inequities.

\section{Conclusions}

The COVID-19 pandemic generated a global crisis, which has disproportionally impacted marginalized communities. Our findings support the assertion that PLWH, by chronically dealing with a potentially deadly disease (i.e. HIV infection), have acquired tools to build resilience, worry less about COVID-19, and experience fewer anxiety symptoms than their HIV-uninfected peers. Nonetheless, the high prevalence of anxiety and worry during the COVID-19 pandemic warrants improved public health efforts for mental health relief. A decline in cocaine use was observed; however, the decline is likely due to reduced availability and higher cost of the drug rather than personal choices. There is much to be learned from exploring how vulnerable communities are affected by the COVID-19 pandemic and the emerging changes on their mental health and substance use patterns. Research is needed to identify psychosocial factors that can improve the ability of marginalized communities to cope with the pandemic by providing strategies to build resilience to better prepare populations before, during, and after broadly impacting crises.

Acknowledgements We thank the MASH Cohort participants, the MASH research team, and C3PNO investigators for their contributions to this work.

Author Contributions Conceptualization: JDM, JAT, SS, PMB, and MKB; Data curation: JAT, IDE, and QL; Formal analysis: IDE and QL; Funding acquisition: SS, PMB, and MKB: Investigation: JDM and JAT; Methodology: JDT, JAT, IDE, MJT, SS, PMB, and MKB; Project administration and supervision: $\mathrm{AC}$ and $\mathrm{MKB}$; Writing —original draft: JDM and JAT; Writing — review and editing: all authors critically revised earlier versions of the manuscript. All authors have read and approved the final version of the manuscript.

Funding The research reported in this publication was supported by the National Institute on Drug Abuse of the National Institutes of Health under Award No. U01-DA040381 and U24-DA044554-04S1. The content is solely the responsibility of the authors and does not necessarily represent the official views of the National Institutes of Health.

Availability of Data Data described in the manuscript, code book, and analytic code will be made available upon request pending application and approval by the principal investigator (baumm@fiu.edu).

\section{Declarations}

Conflict of interest The authors have no conflicts of interest to report.

Consent to Participate All participants provided written informed consent.

Ethics Approval FIU Institutional Review Board.

\section{References}

1. World Health Organization. Mental health and psychosocial considerations during the COVID-19 outbreak. World Health Organization; 2020. Report No.: WHO/2019-nCoV/MentalHealth/2020.1. https://www.who.int/publications/i/item/WHO2019-nCoV-MentalHealth-2020.1.

2. Salari N, Hosseinian-Far A, Jalali R, Vaisi-Raygani A, Rasoulpoor S, Mohammadi M, et al. Prevalence of stress, anxiety, depression among the general population during the COVID-19 pandemic: a systematic review and meta-analysis. Global Health. 2020;16(1):57. https://doi.org/10.1186/ s12992-020-00589-w.

3. Shiau S, Krause KD, Valera P, Swaminathan S, Halkitis PN. The burden of COVID-19 in people living with HIV: a syndemic perspective. AIDS Behav. 2020;24(8):2244-9. https://doi.org/10. 1007/s10461-020-02871-9.

4. Yao H, Chen JH, Xu YF. Patients with mental health disorders in the COVID-19 epidemic. Lancet Psychiatry. 2020;7(4): https:// doi.org/10.1016/S2215-0366(20)30090-0.

5. Pellowski JA, Kalichman SC, Matthews KA, Adler N. A pandemic of the poor: social disadvantage and the U.S. HIV epidemic. Am Psychol. 2013;68(4):197-209. https://doi.org/10.1037/a0032694.

6. Duko B, Ayalew M, Ayano G. The prevalence of alcohol use disorders among people living with HIV/AIDS: a systematic review and meta-analysis. Subst Abuse Treat Prev Policy. 2019;14(1):52. https://doi.org/10.1186/s13011-019-0240-3.

7. Gallant J, Hsue PY, Shreay S, Meyer N. Comorbidities among US patients with prevalent HIV infection-a trend analysis. J Infect Dis. 2017;216(12):1525-33. https://doi.org/10.1093/infdis/jix518.

8. Yang HY, Beymer MR, Suen SC. Chronic disease onset among people living with HIV and AIDS in a large private insurance claims dataset. Sci Rep. 2019;9(1):18514. https://doi.org/10.1038/ s41598-019-54969-3.

9. Amin P, Douaihy A. Substance use disorders in people living with human immunodeficiency virus/AIDS. Nurs Clin N Am. 2018;53(1):57. https://doi.org/10.1016/j.cnur.2017.10.007.

10. De Santis JP, Florom-Smith A, Vermeesch A, Barroso S, DeLeon DA. Motivation, management, and mastery: a theory of resilience in the context of HIV infection. J Am Psychiatr Nurses Assoc. 2013;19(1):36-46. https://doi.org/10.1177/1078390312474096.

11. Norris FH, Tracy M, Galea S. Looking for resilience: understanding the longitudinal trajectories of responses to stress. Soc Sci Med (1982). 2009;68(12):2190-8. https://doi.org/10.1016/j.socsc imed.2009.03.043.

12. Windle G. What is resilience? A review and concept analysis. Rev Clin Gerontol. 2010;21(2):152-69. https://doi.org/10.1017/s0959 259810000420.

13. Diehl M, Hay EL, Chui H. Personal risk and resilience factors in the context of daily stress. Annu Rev Gerontol Geriatr. 2012;32(1):251-74. https://doi.org/10.1891/0198-8794.32.251.

14. Zhang J, Yang Z, Wang X, Li J, Dong LL, Wang FS, et al. The relationship between resilience, anxiety and depression among patients with mild symptoms of COVID-19 in China: a crosssectional study. J Clin Nurs. 2020;29(21-22):4020-9. https://doi. org/10.1111/jocn.15425.

15. Zaami S, Marinelli E, Vari MR. New trends of substance abuse during COVID-19 pandemic: an international perspective. Front Psychiatry. 2020;11:700. https://doi.org/10.3389/fpsyt. 2020. 00700.

16. NIDA. COVID-19: Potential implications for individuals with substance use disorders. National Institute on Drug Abuse website. https://www.drugabuse.gov/about-nida/noras-blog/2020/04/ covid-19-potential-implications-individuals-substance-use-disor ders. April 6, 2020. 
17. Centers for Disease Control and Prevention. HIV surveillance report, 2018 (Updated). 2020. http://www.cdc.gov/hiv/library/ reports/hiv-surveillance.html.

18. Lieb S, Rosenberg R, Arons P, Malow RM, Liberti TM, Maddox LM, et al. Age shift in patterns of injection drug use among the HIV/AIDS population in Miami-Dade County, Florida. Subst Use Misuse. 2006;41(10-12):1623-35. https://doi.org/10.1080/10826 080600847985.

19. Bartholomew TS, Onugha J, Bullock C, Scaramutti C, Patel H, Forrest DW, et al. Baseline prevalence and correlates of HIV and HCV infection among people who inject drugs accessing a syringe services program; Miami, FL. Harm Reduct J. 2020;17(1):40. https://doi.org/10.1186/s12954-020-00385-0.

20. Zarini G, Sales Martinez S, Campa A, Sherman K, Tamargo J, Hernandez Boyer J, et al. Sex differences, cocaine use, and liver fibrosis among African Americans in the Miami Adult Studies on HIV Cohort. J Womens Health. 2020;29(9):1176-83. https://doi. org/10.1089/jwh.2019.7954.

21. Blanco JL, Ambrosioni J, Garcia F, Martinez E, Soriano A, Mallolas J, et al. COVID-19 in patients with HIV: clinical case series. Lancet HIV. 2020;7(5):e314-6. https://doi.org/10.1016/S23523018(20)30111-9.

22. Gervasoni C, Meraviglia P, Riva A, Giacomelli A, Oreni L, Minisci D, et al. Clinical features and outcomes of HIV patients with coronavirus disease 2019. Clin Infect Dis. 2020. https://doi. org/10.1093/cid/ciaa579.

23. Spitzer RL, Kroenke K, Williams JB, Lowe B. A brief measure for assessing generalized anxiety disorder: the GAD-7. Arch Intern Med. 2006;166(10):1092-7. https://doi.org/10.1001/archinte.166. 10.1092 .

24. Ruiz MA, Zamorano E, Garcia-Campayo J, Pardo A, Freire O, Rejas J. Validity of the GAD-7 scale as an outcome measure of disability in patients with generalized anxiety disorders in primary care. J Affect Disorders. 2011;128(3):277-86. https://doi.org/10. 1016/j.jad.2010.07.010.

25. Smith BW, Dalen J, Wiggins K, Tooley E, Christopher P, Bernard $\mathrm{J}$. The brief resilience scale: assessing the ability to bounce back. Int J Behav Med. 2008;15(3):194-200. https://doi.org/10.1080/ 10705500802222972.

26. Windle G, Bennett KM, Noyes J. A methodological review of resilience measurement scales. Health Qual Life Outcomes. 2011;9:8. https://doi.org/10.1186/1477-7525-9-8.

27. Bush K, Kivlahan DR, McDonell MB, Fihn SD, Bradley KA. The AUDIT alcohol consumption questions (AUDIT-C): an effective brief screening test for problem drinking. Ambulatory Care Quality Improvement Project (ACQUIP). Alcohol Use Disorders Identification Test. Arch Intern Med. 1998;158(16):1789-95. https:// doi.org/10.1001/archinte.158.16.1789.

28. Kantamneni N. The impact of the COVID-19 pandemic on marginalized populations in the United States: a research agenda. J Vocat Behav. 2020;119:103439. https://doi.org/10.1016/j.jvb. 2020.103439.

29. Beer L, Tie Y, Padilla M, Shouse RL, Medical Monitoring P. Generalized anxiety disorder symptoms among persons with diagnosed HIV in the United States. AIDS. 2019;33(11):1781-7. https://doi.org/10.1097/QAD.0000000000002286.

30. Twenge JM, Joiner TEUS. Census Bureau-assessed prevalence of anxiety and depressive symptoms in 2019 and during the 2020 COVID-19 pandemic. Depress Anxiety. 2020;37(10):954-6. https://doi.org/10.1002/da.23077.

31. McGowan JA, Sherr L, Rodger AJ, Fisher M, Miners A, Anderson $\mathrm{J}$, et al. Age, time living with diagnosed HIV infection, and self-rated health. HIV Med. 2017;18(2):89-103. https://doi.org/ 10.1111/hiv. 12398.

32. Lyons A, Heywood W. Collective resilience as a protective factor for the mental health and well-being of HIV-positive gay men.
Psychol Sex Orientat Gender Divers. 2016;3(4):473-9. https:// doi.org/10.1037/sgd0000197.

33. Emlet CA, Shiu C, Kim HJ, Fredriksen-Goldsen K. Bouncing back: resilience and mastery among HIV-positive older gay and bisexual men. The Gerontologist. 2017;57(suppl 1):S40-9. https:// doi.org/10.1093/geront/gnw171.

34. Medical Examiners Commission. Drugs identified in deceased persons by Florida Medical Examiners. Florida Department of Law Enforcement; 2020. https://www.fdle.state.fl.us/MEC/ Publications-and-Forms/Documents/Drugs-in-Deceased-Perso ns/2019-Interim-Drug-Report-FINAL.aspx.

35. Crane HM, McCaul ME, Chander G, Hutton H, Nance RM, Delaney JAC, et al. Prevalence and factors associated with hazardous alcohol use among persons living with HIV across the US in the current era of antiretroviral treatment. AIDS Behav. 2017;21(7):1914-25. https://doi.org/10.1007/ s10461-017-1740-7.

36. Ovalle D. Cocaine, meth and weed prices have skyrocketed in Miami: Here's why. Miami Herald; 2020. https://amp.miamiherald.com/news/local/crime/article246014720.html. Accessed 2 Oct 2020.

37. United Nations Office On Drugs And Crime. COVID-19 and the drug supply chain: from production and trafficking to use. Research and Trend Analysis Branch \& UNODC Global Research Network; 2020. https://www.unodc.org/documents/data-and-analy sis/covid/Covid-19-and-drug-supply-chain-Mai2020.pdf.

38. Grant BF, Stinson FS, Dawson DA, Chou SP, Dufour MC, Compton $\mathrm{W}$, et al. Prevalence and co-occurrence of substance use disorders and independent mood and anxiety disorders: results from the national epidemiologic survey on alcohol and related conditions. Alcohol Res Health. 2006;29(2):107-20.

39. Pettinati HM, O'Brien CP, Dundon WD. Current status of cooccurring mood and substance use disorders: a new therapeutic target. Am J Psychiatry. 2013;170(1):23-30. https://doi.org/10. 1176/appi.ajp.2012.12010112.

40. Kessler RC, Chiu WT, Demler O, Merikangas KR, Walters EE. Prevalence, severity, and comorbidity of 12-month DSM-IV disorders in the National Comorbidity Survey Replication. Arch Gen Psychiatry. 2005;62(6):617-27. https://doi.org/10.1001/archpsyc. 62.6.617.

41. Cadet JL. Epigenetics of stress, addiction, and resilience: therapeutic implications. Mol Neurobiol. 2016;53(1):545-60. https:// doi.org/10.1007/s12035-014-9040-y.

42. Ungar M, Theron L. Resilience and mental health: how multisystemic processes contribute to positive outcomes. Lancet Psychiatry. 2020;7(5):441-8. https://doi.org/10.1016/S2215-0366(19) 30434-1.

43. Davydov DM, Stewart R, Ritchie K, Chaudieu I. Resilience and mental health. Clin Psychol Rev. 2010;30(5):479-95. https://doi. org/10.1016/j.cpr.2010.03.003.

44. Chandra A, Acosta JD, Meredith LS, Sanches K, Howard S, Uscher-Pines L, et al. Understanding community resilience in the context of national health security: A literature review: RAND Corporation; 2010. https://www.rand.org/pubs/working_papers/ WR737.html.

45. Chandra A, Acosta J, Howard S, Uscher-Pines L, Williams M, Yeung D, et al. Building community resilience to disasters: a way forward to enhance National Health Security. Rand Health Q. 2011;1(1):6.

46. Moreno C, Wykes T, Galderisi S, Nordentoft M, Crossley N, Jones $\mathrm{N}$, et al. How mental health care should change as a consequence of the COVID-19 pandemic. The Lancet Psychiatry. 2020;7(9):813-24. https://doi.org/10.1016/S2215-0366(20) 30307-2. 
47. Morton MJ, Lurie N. Community resilience and public health practice. Am J Public Health. 2013;103(7):1158-60. https://doi. org/10.2105/AJPH.2013.301354.

48. Dale SK, Bogart LM, Wagner GJ, Galvan FH, Klein DJ. Medical mistrust is related to lower longitudinal medication adherence among African-American males with HIV. J Health Psychol. 2014;21(7):1311-21. https://doi.org/10.1177/1359105314551950.

49. Koch JW. Racial minorities' trust in government and government decisionmakers. Soc Sci Q. 2019;100(1):19-37. https://doi.org/ 10.1111/ssqu. 12548 .

50. Shoff C, Yang T-C. Untangling the associations among distrust, race, and neighborhood social environment: a social disorganization perspective. Soc Sci Med. 2012;74(9):1342-52. https://doi. org/10.1016/j.socscimed.2012.01.012.
51. Centers for Disease Control and Prevention. Monitoring selected national HIV prevention and care objectives by using HIV surveillance data-United States and 6 dependent areas, 2018. HIV Surveillance Supplemental Report [Internet]. 2020 May 2020; 25. http://www.cdc.gov/hiv/library/reports/hiv-surveillance.html.

Publisher's Note Springer Nature remains neutral with regard to jurisdictional claims in published maps and institutional affiliations. 